\title{
Magnetic fractures or reconnection of type II
}

\author{
Gerhard Haerendel \\ Max Planck Institute for extraterrestrial Physics \\ 85748 Garching, Germany \\ email: hae@mpe.mpg.de
}

\begin{abstract}
The importance of reconnection in astrophysics has been widely recognized. It is instrumental in storing and releasing magnetic energy, the latter often in a dramatic fashion. A closely related process, playing in very low beta plasmas, is much less known. It is behind the acceleration of auroral particles in the low-density environment several $1000 \mathrm{~km}$ above the Earth. It involves the appearance of field-parallel voltages in presence of intense field-aligned currents. The underlying physical process is the release of magnetic shear stresses and conversion of the liberated magnetic energy into kinetic energy of the particles creating auroral arcs. In this process, field lines disconnect from the field anchored in the ionosphere and reconnect to other field lines. Because of the stiffness of the magnetic field, the process resembles mechanical fractures. It is typically active in the low-density magnetosphere of planets. However, it can also lead to significant energy conversion with high-energy particle production and subsequent gamma ray emissions in stellar magnetic fields, in particular of compact objects.
\end{abstract}

Keywords. Auroral acceleration, magnetosphere, electric currents, neutron stars

\section{Introduction}

The auroral acceleration process is one of the best explored space plasma processes (Paschmann et al., 2002). Accelerating electric fields with appreciable potential drops parallel to the magnetic field appear in concentrated currents and low densities above the ionosphere. A host of wave processes as well as double layers supporting the electric fields have been identified and related to specific particle distributions. Less well explored are the connections with the high-beta source regions in the outer magnetosphere and near-Earth magnetotail. The energy powering the auroral acceleration process is carried earthward in the Alfvénic wave mode and involves the build-up of magnetic shear stresses. The key issue is that the high-altitude plasma motions and related transverse electric field components are decoupled from those controlled by the collisional ionosphere due to the parallel potential drops, thereby releasing the shear stresses. The author has compared this process with mechanical fractures (Haerendel, 1980; Haerendel, 1988; Haerendel, 1980; Haerendel, 1994; Haerendel, 2007) There are several features shared by mechanical and 'magnetic' fractures: Shear stresses exerted on an elastic elongated medium, a rod or a flux tube, are concentrating in a region of structural weakness; the actual breaking occurs on the molecular or microphysical level; while the fracture propagates spontaneously, a stress release motion is initiated and elastic energy converted into kinetic energy of the elastic medium; subsequently, it is dissipated by some damping mechanism; in case of the aurora by acceleration and heating of particles. This paper deals with these aspects in the context of auroral arcs. A condensed formalism is presented allowing a simple evaluation of the relation between stress application, stress release, energy flux, and field-parallel potential drops, and is also easily adapted to other astrophysical systems. 

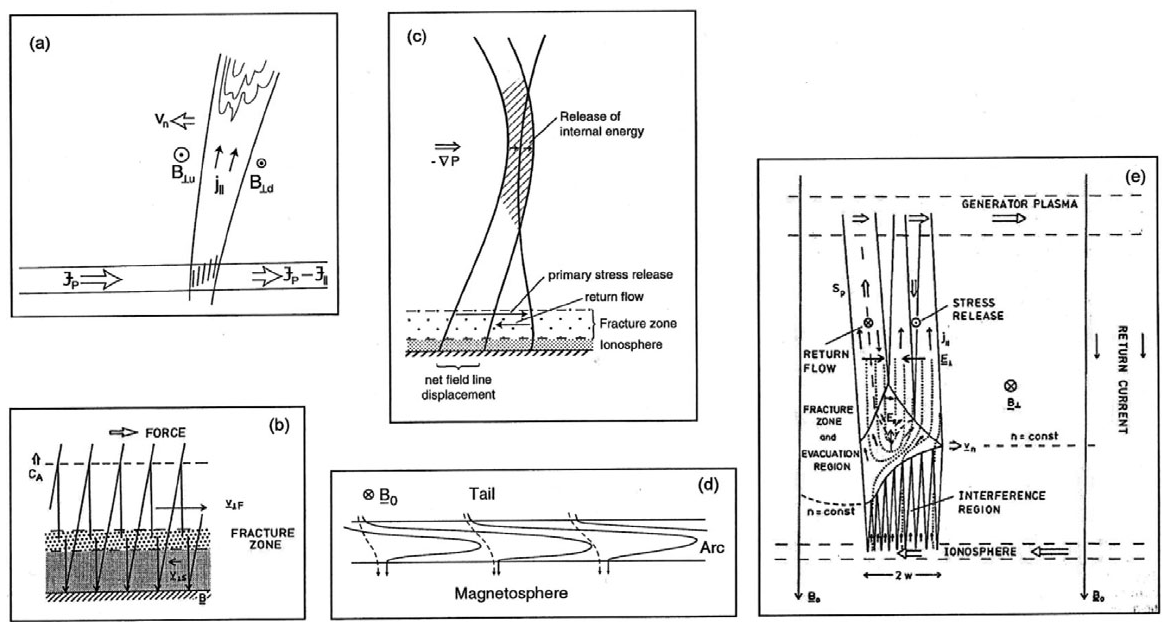

Figure 1. The fracture process or release of the differential shear stresses between two sides of a field-aligned current sheet (1a). Figures (1b) and (1c) show the stress release in the plane of the motion, and (1d) in ground projection in the frame of the propagating arc. The dashed lines in (1d) are the tracks of the ionospheric section. Figure 1e shows oblique Alfvén waves attached to the fracture zone and equipotential contours (dotted lines). (After Haerendel, 1994; Haerendel, 2007; Haerendel, 2009)

\section{The Fracture Process}

Figure 1 illustrates the stress release process in various projections. Figure 1a shows a sheet of upward directed field-aligned current extending normal to the plane of projections for the case of arcs embedded in a wider current system. The sheet current separates a region of highly stressed magnetic field from a less stressed one. It is the differential magnetic energy residing in the shear stresses that is being converted into kinetic energy of the auroral particles. Figure $1 \mathrm{~b}$ shows that previously connected field lines (continuous inclined lines) are separated by the developing fracture zone into an upper magnetospheric and lower ionospheric section, while the attached plasma undergoes opposing stress relief motions. As the decoupling process is initiated somewhere above the ionosphere, the stress relief motions are propagated upward towards the source region of the shear stresses and affect the force balance. This is illustrated in Figure 1c. Since the shear stresses in the source region are being reduced, the plasma is accelerated in the direction of the primary force, here the pressure gradient force. After a while, the stress reduction fades and the plasma slows down. At the same time, the lower section of the magnetospheric part of the flux tube overshoots and is forced back, until the less strained configuration of the field at the rear of the current sheet is reached. This is shown in Figure 1d as a ground projection of the tracks of the magnetospheric as well as the ionospheric field lines in the frame of the arc. When the process is completed, new magnetic connections have been established. The auroral acceleration process is truly a reconnection process albeit in very low- $\beta$ plasma.

Figure 1e contains an attempt to sketch the communication of the magnetic perturbations up and down the field lines during the fracture process. When the leading edge of the fracture zone hits so far unperturbed field lines, transverse motions are induced starting the stress release. The resulting perturbations are propagating in the Alfvén mode towards the source region while the 'fracture' progresses slowly into the current circuit. The large ratio of the two speeds causes the very slight obliqueness of the wave 
fronts. The field changes resulting from the dynamic reaction of the source plasma are again communicated towards the fracture zone. Meanwhile the low-altitude overshoot of the flux tubes is reversed and the field lines are being dragged into the lower shear configuration at the rear of the current sheet including the displacement of the source plasma. The detailed evaluation of Haerendel (1994) and 2007, shows that at least four transit times of an Alfvén wave between fracture zone and source plasma are required for this process. The key point is the near-incompressibility of the magnetic field near the planet (or star), which allows a representation of the electric fields as potential fields. Thus parallel fields are by necessity connected with transverse components and the fieldaligned acceleration process with perpendicular stress relief motions. The dotted lines in Figure 1e depict these mainly U-shaped potential contours. The asymmetry of the transverse potentials between the leading and trailing edges corresponds to the asymmetry in the horizontal excursions in Figure 1d. The 'interference region' between fracture zone and lower ionosphere is probably dominated by Alfvénic turbulence and provides further decoupling (Haerendel, 2007).

There is rich experimental evidence for the U-shaped potentials at several $1000 \mathrm{~km}$ height from satellite crossings and electrostatic double probe measurements (Mozer et al., 1977, Paschmann et al., 2002). Another type of evidence comes from optical observations of auroral ray or fold motions when viewed along the magnetic field. By electron impact the counterflows of the stress release process are imaged on the atmosphere (Davis, 1978). Observations by Haerendel et al. (1996) clearly exhibit also the asymmetry between the leading and trailing edges. Simultaneous radar measurements confirm that the arcs are propagating, i.e. they are not frozen in the plasma frame.

\section{Stress Concentration Mechanisms}

Fractures of a strained elastic medium (e.g. a long rod) develop where stress concentration meets with structural weakness. In the magnetic case, this is achieved by concentrations of the field-aligned current in combination with a low density of the current carrying electrons (Kindel \& Kennel, 1971). Various mechanisms exist creating field-parallel voltages and thus 'breaking' of the magnetic connections. Planetary magnetospheres with their low density and converging magnetic fields are particularly prone to develop such stress concentrations at relatively low altitudes (several $1000 \mathrm{~km}$ in the Earth's case). Knight (1973) and Fridman \& Lemaire (1980) have developed a simple current-voltage relation, which has found rich application in the interpretation of observational data. It is due to the mirror effect creating a dearth of current carriers for a current imposed by the large-scale plasma dynamics. For large mirror ratios it can be simplified as:

$$
\Phi_{\|}=K^{-1} \cdot j_{\|},
$$

where $\mathrm{K}$ is the conductance derived in the original papers for the inverse current-voltage relation.

While Equation 3.1 can be well applied to quasi-stationary arc models like the one shown in Figure 1e, propagating Alfvén waves have to be handled differently. According to Goertz \& Boswell (1979), parallel electric fields exist in the inertial regime if the transverse scales are of the order of the electron inertial length, $\lambda_{e}$. In this case, the parallel and transverse potentials are of the same magnitude:

$$
\Phi_{\|} \approx \Phi_{\perp} \quad \text { for } \quad k_{\perp}^{2} \cdot \lambda_{e}^{2} \geqslant 1
$$

In the much higher densities of stars or other astrophysical systems, the above conditions are hardly realized. However, substantial field-aligned voltages will arise if the current 

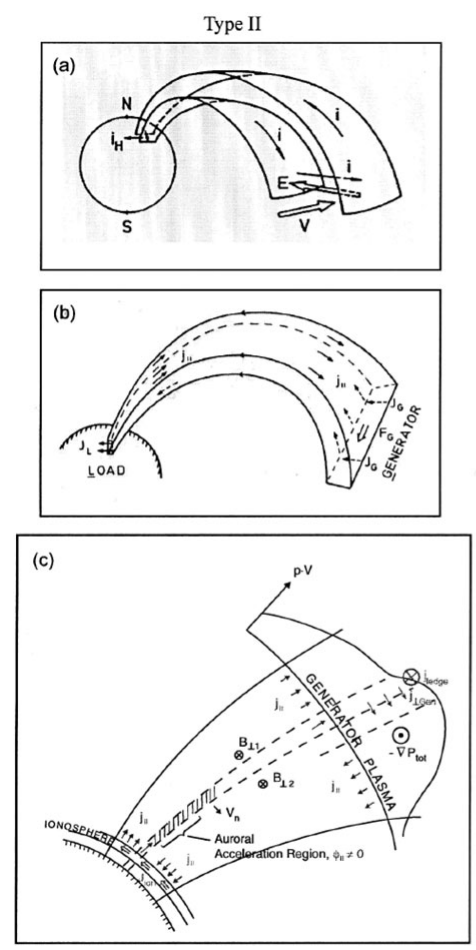
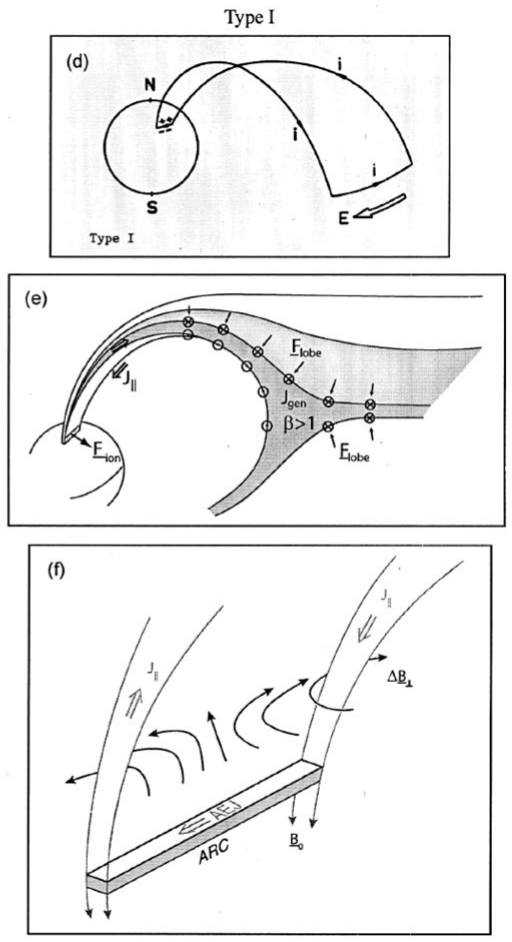

Figure 2. Two current systems after Boström (1964) and associated arcs: Type II with the generator forces acting parallel to the resulting arc, and Type I with the force acting perpendicular to it (Haerendel, 1988; Haerendel, 1994; Haerendel, 2007; Haerendel, 2009).

density exceeds a critical threshold, $j_{c r i t}$, which depends on the electron to ion temperature ratio (Papadopoulos, 1977). One can, for instance, scale the critical drift velocity, $u_{d}$, by the ion thermal speed and a scaling factor, $f$ :

$$
u_{d} \geqslant \frac{j_{\text {crit }}}{e n}=f \cdot v_{t h, i}
$$

$f$ is typically of the order of 10 for the most unstable ion cyclotron waves and $T_{i} \approx T_{e}$ (Treumann \& Baumjohann, 1997).

There is a host of microphysical processes which actually sustain the parallel electric fields, such as pressure gradients, electron inertia, solitary waves associated with ion or electron phase space holes, large-amplitude ion acoustic or ion cyclotron waves, and double layers. Several processes relevant for auroral physics are discussed in detail in Paschmann et al., 2002.

\section{Stress Applications and Release}

One of the pioneering contributions to auroral physics was the identification of the two basic current circuits underlying the interaction of the outer realms of the magnetosphere with the ionosphere and, for that matter, also with the auroral energy conversion regions (Boström, 1964). They are distinguished by the orientation of the driving forces with respect to the dissipation regions. Figure 2 shows the two principal current systems, referred to as Type II and Type I, the respective connections between source region and acting force, the current closure regions in the ionosphere, and the resulting arcs. The Type II system, displayed in Figure 2 a-c, are typical for the convective flows of the 


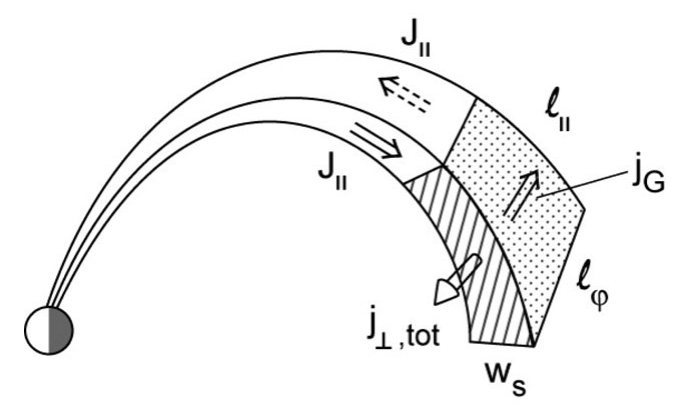

Figure 3. A high- $\beta$ plasma layer as source of field-aligned currents, after Haerendel (2009).

magnetospheric and ionospheric plasmas, for instance along the auroral oval. They are driven by pressure gradient forces. Figure $2 \mathrm{c}$ shows the frequent situation of auroral arcs imbedded in the larger convection channel. These arcs carry only a fraction of the total current flowing between the generator region and the ionosphere in the normal direction to the arc. Figures 2d-f deal with the situation encountered at the boundary between the tail (polar cap) and the outer magnetosphere. The solar wind, by compressing the magnetotail, exerts a force on the outer more dipolar magnetospheric field. In contrast to the Type II case, the currents are flowing along the auroral arc, which is a region of enhanced conductivity. The downward transported shear stresses act in the normal direction to the arc.

\section{Key Relations}

The author has cast his concept of magnetic fractures into a small set of analytical relations, which also allow application to other than planetary environments (Haerendel, 2007; Haerendel, 2009). We assume a quasi-stationary situation, in which the arc system exists longer than the four Alfvénic transit times underlying the model of Figure 1. Quasi-stationarity implies a matching between the electromagnetic energy inflow from the generator, the Poynting flux, $S$, and the conversion rate into particle energy, $\dot{W}$, whereby any ohmic losses by the closure currents are being neglected. With the length of the field line, $L_{\text {par }}$, between generator and fracture zone and the respective Alfvénic transit time, $\tau_{A}$, one can define an integral wave impedance

$$
R_{w}=\frac{\mu_{0} L_{\|}}{\tau_{A}}
$$

and with the sheet current density, $J_{\|, a r c}$, the Poynting flux becomes:

$$
S_{a r c}=R_{w} \cdot J_{\|, a r c}^{2}=\dot{W}_{a r c}
$$

In Figure 3, a current wedge of Type I is being displayed. The various dimensions characterizing the current system are the field-parallel and longitudinal extensions, $l_{\|, G e n}$ and $l_{\phi, G e n}$, and the projection of the latter into the fracture zone, $l_{a r c}$. Following the derivation in Haerendel (2009), one gets:

$$
J_{\|, a r c}=\frac{l_{\varphi, G e n}}{l_{\text {div }}} \cdot \frac{l_{\|, G e n}}{l_{\text {arc }}} \cdot \frac{\beta_{G e n}}{2} \cdot \frac{B_{G e n}}{\mu_{0}}
$$

The ratio, $l_{\varphi, G e n} / l_{d i v} \approx 0.1$ expresses that only a fraction of the current flowing in the generator layer is actually diverted towards the star. This means that most of the force acting on the generator plasma is balanced by magnetic normal stresses and only a small 
fraction by shear stresses transported towards low altitudes. The comparison of theory and data in the auroral context shows that typical auroral current densities and energy flows require $\beta_{G e n} \geqslant 1$. Furthermore, it is advantageous to decompose the second factor in Equation 5.3, since there is little a priori knowledge of the length of the arc:

$$
\frac{l_{\|, G e n}}{l_{\text {arc }}}=\frac{l_{\|, G e n}}{l_{\varphi, \text { Gen }}} \cdot \frac{l_{\varphi, \text { Gen }}}{l_{\text {arc }}} \approx \frac{l_{\|, G e n}}{l_{\varphi, G e n}} \cdot \sqrt{\frac{B_{\text {arc }}}{B_{\text {Gen }}}}
$$

This way, current or Poynting flux near the fracture zone are entirely characterized by the plasma and field parameters and spatial dimensions of the generator and the magnetic field at the energy conversion level.

The accelerating parallel voltage, $\Phi_{\|}$, depends on the arc width, $w_{\text {arc }}$, which is either determined by the inverse conductance, $K^{-1}$ (Equation 3.1), or the critical current density, $j_{\text {crit }}$ :

$$
\Phi_{\|}=\dot{W}_{a r c} \cdot \frac{w_{a r c}}{J_{\|, a r c}} \quad \text { and } \quad \frac{J_{\|, a r c}}{w_{a r c}} \cong j_{c r i t}
$$

The last expression is better suited for the application to astrophysical systems.

\section{Why Reconnection of Type II?}

We have demonstrated that the auroral acceleration process involves reconnection, but the concept of magnetic fractures better describes what is happening. Since neither magnetic fractures nor their characterization as a reconnection process has yet found wide acceptance among auroral researchers, we will close this paper with a short list of features of this process justifying the designation 'reconnection of type II':

- In the decoupling process, the magnetospheric field undergoes new connections with the ionospheric field.

- There is a dissipation region, the fracture zone, corresponding to the so-called diffusion region in Type I reconnection.

- There is also a wave region, oblique quasi-stationary or fast propagating Alfvén waves, transferring momentum to the ambient plasma and channeling the energy flux.

- Type II, because momentum and energy inflow are widely separated from the dissipation region.

\section{References}

Boström, R. 1964, J. Geophys. Res., 69, 4893, doi:10.1029/JZ69i023p04983

Davis, T. N. 1978, Space Sci. Revs, 22, 77

Fridman, M. \& Lemaire, J. 1980, J. Geophys. Res., 85, 664

Goertz, C. K. \& Boswell, R. W. 1979, J. Geophys. Res., 84, 7239

Haerendel, G. 1980, ESA Journal, 4, 197

Haerendel, G. 1988, ESA-SP 285, Vol.1, 37, Proc. ESA Symp. Varenna/Italy

Haerendel, G. 1994, ApJS, 90, 765

Haerendel, G. et al. 1996, J. Atmos. Sol. Terr. Phys., 58, 71

Haerendel, G. 2007, J. Geophys. Res., 112, A09214, doi:10.1029/2007JA012378

Haerendel, G. 2009, J. Geophys. Res., 114, A06214, doi:10.1029/2009JA014139

Kindel, J. M. \& Kennel, C. F. 1971, J. Geophys. Res., 76, 3055

Knight, S. 1973, Planet. Space Sci., 21, 741

Mozer, F. et al. 1977, Phys. Rev. Lett., 38, 292

Papadopoulos, K. 1977, Rev. Geophys. Spce Sci., 15, 113

Paschmann, G., Haaland, S., \& Treumann, R. (eds.) 2002, Space Sci. Revs, 103, Nos. 1-4

Treumann, R. \& Baumjohann, W. 1997, Advanced Plasma Physics, Imperial College Press 